content was remarkably elevated.

In order to clarify the above phenomenon, the human sera were stored aseptically at $37^{\circ} \mathrm{C}$ and their fibrinolytic activities were measured periodically for long periods. This experiment revealed that the activities increased until 26th day when the reached at the highest value, and then decreased gradually. It was presumed that, when the blood was retained in the subdural space presumed that, when the blood was retained in the subdural space for a certain period, the caspule was formed, from which minor bleeding were repeated within capsule, and thus the fibrinolytic activities in the hematoma contents were kept high.

4) In one patient with subdural hematoma, a serial measurement of the fibrinolytic activities of cerebrospinal fluid and of the plasma were undertaken from 15 th post-traumatic day to $63 \mathrm{rd}$ day (the day of the operation). The activities of the cerebrospinal fluid and plasma were noted to be high until 35 th and 21 st post-traumatic day, respectively. The elevation of the fibrinolytic activities during these period was considered due to repeated bleeding from the wellformed hematoma capsule.

\title{
148. Non-bleeding Treatment for the Chronic Subdural Haematoma
}

\author{
The Preliminary Report
}

\author{
Jiro Suzuki, Norio Harada, Ryungcham KwaK, Masaaki TakahashI, \\ Hiromichi Aimara and Sadao TAKase \\ Department of Neurosurgery, Institute of Brain Diseases, \\ Tohoku University School of Medicine
}

Eight cases of chronic subdural haematoma including two severe patients have been treated with non-bleeding therapy by osmotherapy chiefly using $20 \%$ Mannitol.

In the all cases the content of haematoma was disappeared or extremely decreased by the treatment for 1 month to 4 months.

By the osmotherapy, the change of character, mental disturbances and other complications were not happened at the following up study from 3 months to 15 months after their discharge.

The thinking process to the performance of this treatment and healing mechanism of the haematoma were discussed. 